Psychother Psychosom 2018;87:240-242

DOI: $10.1159 / 000489498$

\title{
The Efficacy of a Group CBT Relapse Prevention Program for Remitted Anxiety Disorder Patients Who Discontinue Antidepressant Medication: A Randomized Controlled Trial
}

\author{
Willemijn D. Scholten ${ }^{\mathrm{a}, \mathrm{b}}$ Neeltje M. Batelaan ${ }^{\mathrm{a}, \mathrm{b}}$ \\ Patricia van Oppen ${ }^{\mathrm{a}, \mathrm{b}}$ Johannes H. Smit ${ }^{\mathrm{a}, \mathrm{b}}$ \\ Adriaan W. Hoogendoorn ${ }^{\mathrm{a}, \mathrm{b}}$ Harold J.G.M. van Megen ${ }^{\mathrm{c}}$ \\ Daniëlle C. Cath ${ }^{\mathrm{d}-\mathrm{g}}$ Anton J.L.M. van Balkom ${ }^{\mathrm{a}, \mathrm{b}}$ \\ a Department of Psychiatry, GGZ ingest, Amsterdam,

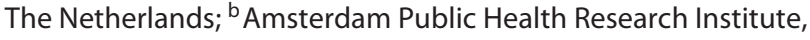 \\ VU University Medical Center, Amsterdam, The Netherlands; \\ 'Innova Research Centre, Mental Health Care Institute, \\ GGZ Centraal, Ermelo, The Netherlands; ${ }^{\mathrm{d}}$ Altrecht Academic

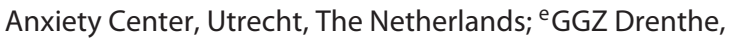 \\ Assen, The Netherlands; ${ }^{\mathrm{f}}$ Department of Clinical Psychology, \\ Utrecht University, Utrecht, The Netherlands; ${ }^{9}$ Department of \\ Psychiatry, University Medical Center Groningen, Groningen, \\ The Netherlands
}

In the treatment of anxiety disorders, antidepressants (AD) are regarded as first-line pharmacological evidence-based treatment $[1,2]$. Based on consensus, guidelines advise to discontinue AD 6-24 months after remission. However, recurrence rates following discontinuation are high. A recent meta-analysis showed that, in the medium term, continuing $\mathrm{AD}$ results in lower recurrence rates (16\%) than discontinuing AD (36\%) [3]. Strategies to prevent recurrence are needed, but lacking.

The aim of this multicenter, randomized controlled trial (Dutch Trial Register NTR2246) was to examine the effect of a cognitive behavioral therapy (CBT) relapse prevention group after discontinuation of $\mathrm{AD}$, compared to "discontinuation as usual" in patients with remitted anxiety disorders. The research protocol was accredited by the Ethics Committee of the VU University Medical Center, and written informed consent was obtained from all participants. Eligible patients ( $>18$ years) (i) used AD but were willing to discontinue, (ii) had no current anxiety or depressive disorder, and (iii) had a history of an anxiety disorder for which they took $\mathrm{AD}$ (panic disorder with or without agoraphobia, agoraphobia, social phobia or generalized anxiety disorder). Participants were recruited from 4 outpatient clinics and through media advertisement (data collection from August 2010 to November 2014). Block randomization was used, stratified per location. Neither patients nor therapists were blinded. The "CBT group discontinuation" consisted of 8 group sessions of relapse prevention, targeting vulnerability factors and discontinuation symptoms. In the "control con-

\section{KARGER}

E-Mail karger@karger.com www.karger.com/pps
2018 The Author(s)

Published by S. Karger AG, Basel

Karger

Open access

This article is licensed under the Creative Commons AttributionNonCommercial-NoDerivatives 4.0 International License (CC BYNC-ND) (http://www.karger.com/Services/OpenAccessLicense). Usage and distribution for commercial purposes as well as any distribution of modified material requires written permir dition," discontinuation without CBT was guided by psychiatrists in individual sessions. AD were tapered within 4 months according to a fixed schedule. Primary outcome measures were (time to) recurrence of: (i) the previous anxiety disorder, (ii) re-/occurrence of any anxiety disorder, and (iii) re-/occurrence of any anxiety disorder or major depressive disorder (MDD). Diagnostic status was verified at baseline and at $4,7,10,13$, and 16 months with the Structured Clinical Interview of Axis I Disorders (SCID-I) [4]. As a secondary outcome, the success rate of discontinuation of $\mathrm{AD}$ was added. To detect an effect size of Cohen $d=0.5$, estimating attrition to be $25 \%$ and considering the multisite design, 109 participants had to be included in each arm. After 7 months (when 87 patients were included), an unplanned interim analysis was conducted by an Independent Data Monitoring Committee, because of the clinical impression that many patients had relapsed already, and more recurrences were expected over time. Inclusion was stopped prematurely for ethical reasons and lack of effect (futility), though assessments of included participants continued until 16 months. Intention-to-treat analyses were conducted to test the effect of the intervention, using the fitted proportional hazard model to estimate "survival without recurrence." Completer analyses, including all participants with complete assessments on recurrence and $\mathrm{AD}$ use, using $\chi^{2}$ statistics were conducted to study recurrence related to discontinuation of $\mathrm{AD}$.

Ninety patients were screened and 87 were randomized. In total, 73 participants started the allocated intervention. Of all participants $69 \%$ had tried to discontinue AD before. No significant differences in baseline characteristics emerged. The Cox regression model showed that over 16 months, CBT was not effective in preventing "recurrence of the previous anxiety disorder" $(\mathrm{HR}=$ $1.042,95 \% \mathrm{CI}=0.526-2.063)$, "re-/occurrence of any anxiety disorder" ( $\mathrm{HR}=0.925,95 \% \mathrm{CI}=0.519-1.648)$ or "re-/occurrence of any anxiety disorder or $\mathrm{MDD}(\mathrm{HR}=0.925,95 \% \mathrm{CI}=0.537-1.594)$. Cumulative recurrence rates were up to $67 \%$, as shown in Figure 1. Despite guidance offered, only $37 \%(n=26)$ of the total group of participants with "complete assessments" $(n=71)$ succeeded in discontinuing $\mathrm{AD}$, with no significant difference between the CBT group and the control group ( 41 vs. $32 \%, \chi^{2}=0.584, p=0.45$ ). Only $28 \%(n=20)$ succeeded in discontinuation of $\mathrm{AD}$ without recurrence, which was the ultimate goal of the intervention. One male participant committed suicide, which was regarded as a serious adverse event. The suicide was reported to the Ethics Committee and the Health Care Inspectorate.

CBT did not protect against recurrence in patients who wanted to discontinue $\mathrm{AD}$. No comparable studies had been conducted previously, except for studies on depression, where the effect of a psychological (mindfulness) intervention after stopping $\mathrm{AD}$ was inconclusive $[5,6]$. Our finding may be partly due to the timing of the CBT, i.e., simultaneously with the discontinuation period, while most recurrences occurred in the period after tapering. The right timing of CBT is crucial [7]. The recurrence rates found in
Willemijn D. Scholten

Department of Psychiatry, Amsterdam Public Health Research Institute VU University Medical Center, A.J. Ernststraat 1187 NL-1081 HL Amsterdam (The Netherlands)

E-Mail w.scholten@ggzingeest.nl 


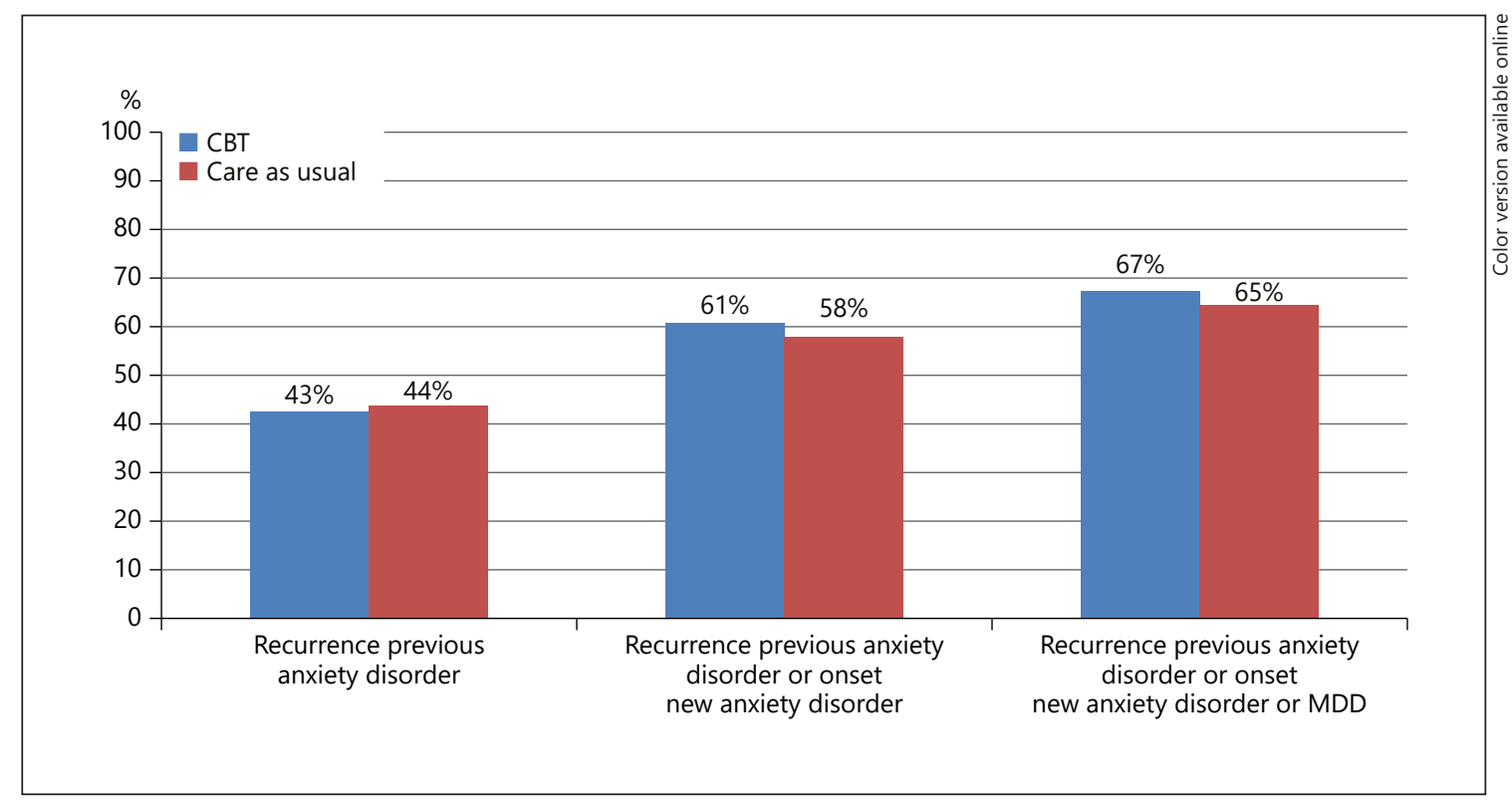

Fig. 1. Predicted recurrence rates (\%) within 16 months.

our study are somewhat higher than expected from previous studies and substantially increase when onset of other anxiety disorders or MDD is included. Furthermore, only $37 \%$ succeeded in discontinuing $\mathrm{AD}$, which is in contrast with higher successful discontinuation rates reported in other CBT and mindfulness studies. The discrepancy may be due to our vulnerable sample, in which the majority had unsuccessfully tried to discontinue before.

There are some limitations. First, the trial was stopped prematurely for ethical reasons and for futility. However, a conditional power calculation showed that results were highly unlikely to change if the trial was taken to completion. Second, withdrawal syndromes, according to Chouinard and Chouinard [8] and Fava et al. [9] were not assessed. Therefore, specific withdrawal syndromes may have been misidentified as recurrence or the occurrence of new disorders. Post hoc analyses showed that directly after the 4-month discontinuation period, recurrence rates were already high (22\% recurrence of the same anxiety disorder; $41 \%$ anxiety disorder or MDD). In this phase, return of original symptoms or occurrence of new disorders might also be considered as persistent postwithdrawal disorders. Postwithdrawal syndromes are the result of an underlying pharmacological mechanism instead of the natural course of a disorder. Third, lack of power hampered the assessment of the true effect of the CBT on recurrence, because only $37 \%$ of the participants discontinued AD completely.

An important clinical implication of this study is that the guidelines' recommendation to discontinue $\mathrm{AD}$ was neither feasible nor effective in our sample. Before the start of pharmacological treatment, patients need to be informed about difficulties associated with discontinuing $\mathrm{AD}$, and the high risk of recurrence or withdrawal symptoms following discontinuation [10].

Further details about the study results are presented in the online supplementary material (for all online suppl. material, see www.karger.com/doi/10.1159/000489498).

\section{Acknowledgments}

We are most grateful to the participants, outpatient clinics and therapists who participated in this study. This study was supported by ZonMw grant No. 171001008 .

\section{Author Contribution}

Each author has sufficiently contributed to the conception and design, interpretation of the data, revising it critically, and has approved of the final version of the manuscript.

\section{Disclosure Statement}

Willemijn Scholten, Neeltje Batelaan, Patricia van Oppen, Jan Smit, Adriaan Hoogendoorn, Harold van Megen, Daniëlle Cath, and Anton van Balkom have no personal affiliations or financial relationship with any commercial interest to disclose relative to this article.

\section{References}

1 Baldwin DS, Anderson IM, Nutt DJ, Allgulander C, Bandelow B, den Boer JA, Christmas DM, Davies S, Fineberg N, Lidbetter N, Malizia A, McCrone P, Nabarro D, O'Neill C, Scott J, van der Wee N, Wittchen HU: Evidence-based pharmacological treatment of anxiety disorders, posttraumatic stress disorder and obsessive-compulsive disorder: a revision of the 2005 guidelines from the British Association for Psychopharmacology. J Psychopharmacol 2014;28:403-439.

-2 Bandelow B, Zohar J, Hollander E, Kasper S, Moller HJ, Zohar J, et al: World Federation of Societies of Biological Psychiatry (WFSBP) guidelines for the pharmacological treatment of anxiety, obsessive-compulsive and post-traumatic stress disorders - first revision. World J Biol Psychiatry $2008 ; 9: 248-312$. 
3 Batelaan NM, Bosman RC, Muntingh A, Scholten WD, Huijbregts KM, van Balkom AJLM: Risk of relapse after antidepressant discontinuation in anxiety disorders, obsessive-compulsive disorder, and post-traumatic stress disorder: systematic review and meta-analysis of relapse prevention trials. BMJ 2017;358:j3927.

4 First MB, Spitzer RL, Gibbon GM, Williams GB: Structured Clinical Interview for DSM-IV-TR Axis Disorders, Research Version, Patient Edition. New York, Biometrics Research, 2002.

5 Huijbers MJ, Spinhoven P, Spijker J, Ruhe HG, van Schaik DJ, van Oppen P, Nolen WA, Ormel J, Kuyken W, van der Wilt GJ, Blom MB, Schene AH, Rogier A, Donders T, Speckens AE: Discontinuation of antidepressant medication after mindfulness-based cognitive therapy for recurrent depression: randomised controlled non-inferiority trial. $\mathrm{Br} \mathrm{J}$ Psychiatry 2016;208:366-373.
6 Piet J, Hougaard E: The effect of mindfulness-based cognitive therapy for prevention of relapse in recurrent major depressive disorder: a systematic review and meta-analysis. Clin Psychol Rev 2011;31:1032-1040.

7 Belaise C, Gatti A, Chouinard VA, Chouinard G: Persistent postwithdrawal disorders induced by paroxetine, a selective serotonin reuptake inhibitor, and treated with specific cognitive behavioral therapy. Psychother Psychosom 2014;83:247-248.

8 Chouinard G, Chouinard VA: New classification of selective serotonin reuptake inhibitor withdrawal. Psychother Psychosom 2015;84:63-71.

9 Fava GA, Gatti A, Belaise C, Guidi J, Offidani E: Withdrawal symptoms after selective serotonin reuptake inhibitor discontinuation: a systematic review. Psychother Psychosom 2015;84:72-81.

10 Fava GA: Rational use of antidepressant drugs. Psychother Psychosom 2014;83:197-204. 